\title{
Multiple micronutrient supplementation improves growth and reduces the risk of anemia among infants in Gaza Strip, Palestine: a prospective randomized community trial
}

\author{
Ali Albelbeisi ${ }^{1 *}$ D, Zalilah Mohd Shariff ${ }^{1}$, Chan Yoke Mun, Hejar Abdul Rahman² and Yehia Abed ${ }^{3}$
}

\begin{abstract}
Background: Infants and young children 6-24 months of age are the most nutritionally vulnerable groups, as their needs for vitamins and minerals are high relative to the amount of food they consume. This study determines the effect of Micronutrient Powder Supplementation (MNP) on growth and hemoglobin of infants in Gaza Strip, Palestine.

Method: This was a two-arm parallel-group randomized controlled trial conducted in two health care clinics of the United Nations Relief and Work Agency (UNRWA) at the Middle Area governorate of Gaza Strip, Palestine. A total of 200 healthy infants aged 6-month-old were recruited and randomized to receive 3 sachets/week of MNP for 12 months alongside with the National Micronutrient Supplement (NMS) $(n=100)$ or NMS alone $(n=100)$. Weight, length, blood hemoglobin, and dietary intakes were measured at 6, 9, 12, 15, 18, and 21 (3 months after the end of intervention) months of age. Analysis was by intention to treat.

Results: The experimental group had a higher concentration of hemoglobin at 12 and 15 months than did the control group, and a significant difference $(p<0.05)$ was observed at 15 months only. Significantly greater weight, length, weight-for-age, length-for-age, and weight-for-length z-scores were observed in the experimental group than that in the control group at 12 and 15 months $(p<0.05)$. The prevalence of anemia (18\% vs. 52\%) and stunting (1\% vs. $11 \%)$ were higher in the control than the experimental group, respectively. After controlling for sex, similar findings were reported.

Conclusions: Addition of MNP to the existing NMS program improved the nutritional status of young children. The potential benefits of MNP supplementation on physical growth and hemoglobin should be considered in the existing NMS program.
\end{abstract}

Trial registration: ISRCTN57594793; Date of registration: 20-03-2018 (Retrospectively registered).

Keywords: Micronutrient supplementation, Child nutritional status, Physical growth, Hemoglobin, Palestine

\footnotetext{
* Correspondence: Alialbelbeisi@gmail.com

${ }^{1}$ Department of Nutrition and Dietetics, Faculty of Medicine and Health Sciences, University Putra Malaysia, Seri Kembangan, Malaysia

Full list of author information is available at the end of the article
}

(c) The Author(s). 2020 Open Access This article is licensed under a Creative Commons Attribution 4.0 International License, which permits use, sharing, adaptation, distribution and reproduction in any medium or format, as long as you give appropriate credit to the original author(s) and the source, provide a link to the Creative Commons licence, and indicate if changes were made. The images or other third party material in this article are included in the article's Creative Commons licence, unless indicated otherwise in a credit line to the material. If material is not included in the article's Creative Commons licence and your intended use is not permitted by statutory regulation or exceeds the permitted use, you will need to obtain permission directly from the copyright holder. To view a copy of this licence, visit http://creativecommons.org/licenses/by/4.0/ The Creative Commons Public Domain Dedication waiver (http://creativecommons.org/publicdomain/zero/1.0/) applies to the data made available in this article, unless otherwise stated in a credit line to the data. 


\section{Introduction}

Micronutrient deficiencies are reported as a global public health problem affecting 2 billion people in the developed and developing countries [1]. Deficiency of vitamins and minerals acts as exacerbating factors in chronic diseases, greatly impacting morbidity, mortality, and quality of life [2]. There is no global estimate of micronutrient deficiencies for under-two children, however, it has been reported that 190 and 293 million preschool children have vitamin A deficiency and anemia, respectively [3, 4]. In 2013, the Palestinian Micronutrient Survey (PMS) reported that vitamin A status was sufficient among $27 \%$ of under-five children only, and severe deficiency was more prevalent (36.8\%) among Gaza Strip children [5] than those in the West bank. Moreover, the overall occurrence of anemia amongst preschool children in Gaza Strip was 59.7\%, and the prevalence of mild and moderate anemia was 46.5, and $13.5 \%$, respectively [6].

The main contributors to micronutrient deficiencies are lack of dietary diversity, poor bioavailability of minerals in plant foods, high incidence of infectious diseases, and increased physiological demands [7, 8]. Furthermore, deficiencies of vitamin A, iron, zinc, and iodine are common consequences of the cereal- and plantbased complementary foods typically introduced to infants and children in developing countries [9]. These foods are often low in energy and poor sources of bioavailable micronutrients particularly zinc and iron due to presence of phytate [8].

Micronutrient supplementation and food fortification have been shown to be the most cost-effective approaches to reduce micronutrient deficiencies among children [10]. Although the benefits of supplementation with single or multiple micronutrients are well-recognized, implementation has been hindered by poor compliance and adherence to the dosage regimens, inadequate coverage and supply, and potential side effects and safety concerns $[11,12]$. In response to these constraints, at home food fortification with micronutrient powders (MNP) was promoted as a novel method to deliver micronutrients with foods. Several studies [13-15] found that MNP is effective in treating anemia and improving biochemical profile of micronutrients among infants and enhanced tolerability. Promising results were also found in recent studies using MNP with different combinations of micronutrients [16-18]. The use of MNP has obtained publicity due to ease of use and low cost [19] and it contains a lipid-encapsulated coating which prevents iron from dissolving directly into food and hence it does not change the color or taste of food or produce iron-related side effects [20]. MNP is a single dose packet of powder containing vital micronutrients that can be mixed onto any semisolid food at home to enrich the food with the essential vitamins and minerals [21].
Although the Palestinian Ministry of Health $(\mathrm{MOH})$ has implemented the National Micronutrient Supplementation program (NMS) since 2001 to improve the nutritional status of under-two children [22], anemia and growth retardation remain public health concerns in Gaza Strip. Radi et al., (2009) reported that 72.8, 34.3, 31.4 , and $31.45 \%$ of children under two in Gaza city were anemic, wasted, stunted, and underweight, respectively [23]. This study was conducted to determine the effect of micronutrient supplement in powder form on physical growth and hemoglobin concentration of infants aged 6 months.

\section{Methods}

\section{Study design and participants}

This randomized controlled trial was conducted in two health care clinics of the UNRWA at the middle area governorate of Gaza Strip, Palestine. The Middle Area governorate has the highest percentage (86.5\%) of refugees in the Gaza Strip, with a low socioeconomic status $[24,25]$. The study was approved by the Helsinki Ethics Committee of Gaza Strip, as well as the Ethics Committee for Research Involving Human Subjects (JKEUPM) of the Faculty of Medicine and Health Sciences, Universiti Putra Malaysia. Permission to conduct the study in the UNRWA clinics was obtained from the Health Affairs Center of the UNRWA in Gaza Strip. This trial was carried out from October 2015 to January 2017.

Sample size was calculated to detect a $0.7 \mathrm{~cm}$ difference in mean length between the experimental and control groups. Allowing a type I error level of 5\% with power of $90 \%$ and assuming a dropout rate of $30 \%, 100$ infants were required for each group. The total number of infants aged 5 months \pm 2 weeks at the selected two clinics were 415. All infants were screened according to the study selection criteria ((healthy e.g. no physical disabilities or diseases, normal z scores $(>-2$ to $<+2$ $\mathrm{SD}$ ) of weight-for-age (WAZ), length-for-age (LAZ), and head circumference-for-age (HCZ), normal birth weight ( $\geq 2.5$ to $\leq 4 \mathrm{~kg}$ ), appropriate for gestational age (AGA), non-anemic, and breastfed for more than 4 months). Upon screening, a total of 300 infants were eligible for inclusion, and then infants' parents were invited to give consent for their infants' participation. They were informed about the purpose of the study, and only infants of parents who agreed with informed consent were included. Accordingly, 200 out of 300 eligible infants (108 males, 92 females) were randomly selected and allocated equally into two groups (Fig. 1).

Infants were randomly assigned to receive either the MNP with NMS or NMS only. MNP (Mix me ${ }^{\mathrm{mm}}$ ) was produced by DSM Nutritional Products Europe, Switzerland. Random allocation was performed with 


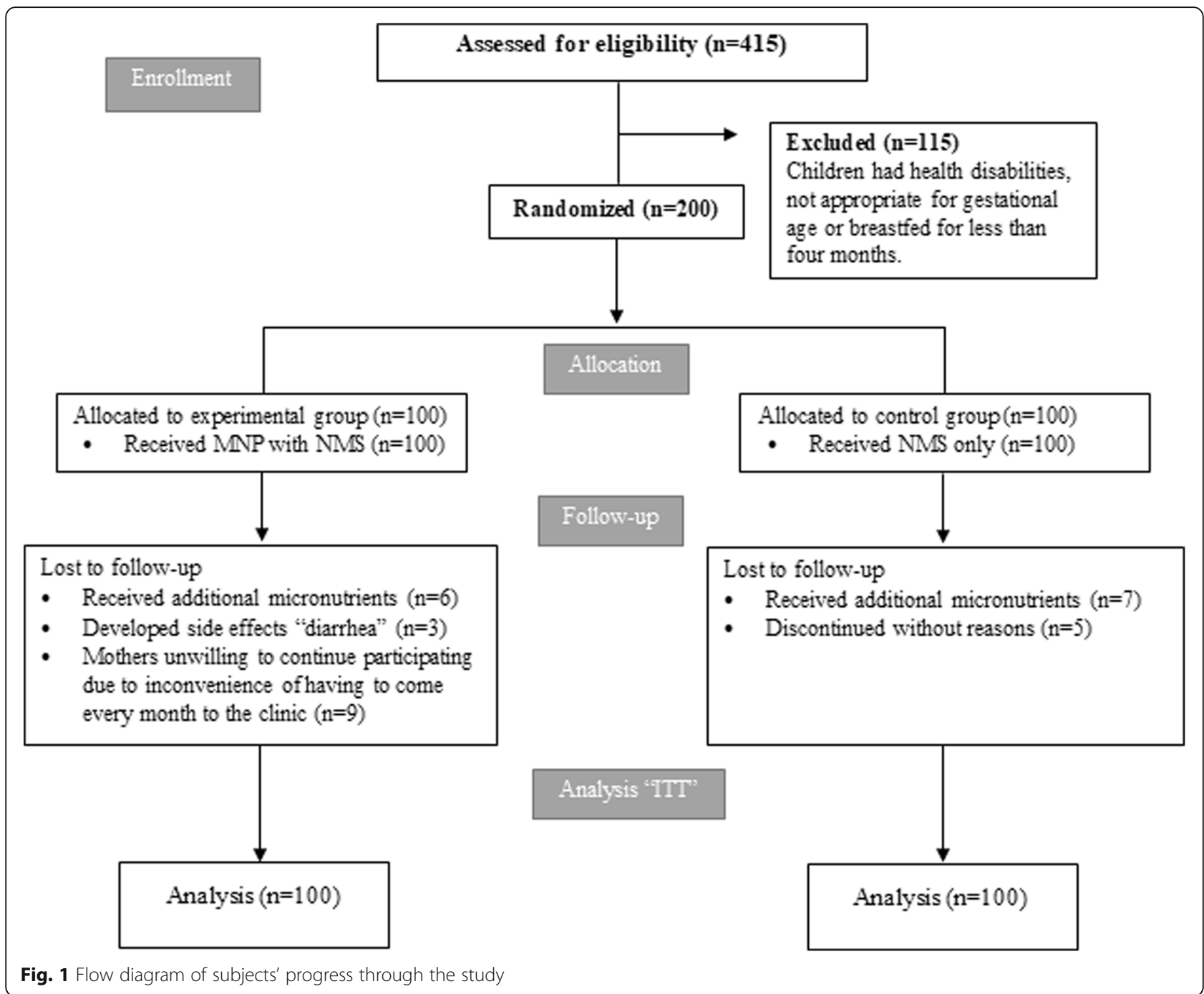

sealed opaque envelopes. Infant's mother was asked to draw an envelope containing the allocation group number ( 1 for the experimental group and 2 for the control group). The study protocol scheduled administration of MNP for 12 months as 3 sachets per week (every other day). MNP were distributed to mothers monthly by two trained research assistants and given as one-dose sachets. MNP was packaged in a paper/aluminum/ polyethylene pouch with lot number and internal batch number printed on the package. Administration of MNP started immediately after baseline assessment. The NMS (Vitamin A, iron, and vitamin D) was provided by the UNRWA as part of the National Nutrition Supplementation program. Micronutrient composition and dosage of MNP and NMS are shown in Tables 1 and 2, respectively. The dose of vitamins and minerals was in accordance to the recommendation by UNICEF [29].

\section{Measurements}

\section{Primary and secondary outcomes}

The primary outcomes were blood hemoglobin and growth parameters such as weight-for-age, length-forage, and weight-for-length. Anemia was defined as mild $(10 \mathrm{~g} / \mathrm{dl} \leq \mathrm{Hb}<11 \mathrm{~g} / \mathrm{dl})$, moderate $(7 \mathrm{~g} / \mathrm{dl} \leq \mathrm{Hb}<$ $10 \mathrm{~g} / \mathrm{dl})$, and severe $(\mathrm{Hb}<7 \mathrm{~g} / \mathrm{dl})$. Underweight was defined as a weight-for-age $\mathrm{z}$ score $<-2.0$; stunting was defined as a length-for-age $\mathrm{z}$ score $<-2 \cdot 0$; and wasting was defined as a weight-for-length $\mathrm{z}$ score $<$ -2.0. Energy and micronutrients intakes (iron, vitamin $\mathrm{A}$, and vitamin D) as well as feeding practices were assessed as secondary outcomes and adequate intake of each dietary element was defined as greater or equal to $100 \%$ Estimated Average Requirements (EAR). Other measures such as demographic and socioeconomic data were also assessed. 
Table 1 Composition of the study supplement (MNP)

\begin{tabular}{ll}
\hline Micronutrient & Composition \\
\hline Vitamin A & $400 \mu \mathrm{g}$ \\
Vitamin C & $30 \mathrm{mg}$ \\
Vitamin D & $5 \mu \mathrm{g}$ \\
Vitamin E & $5 \mathrm{mg}$-TE \\
Vitamin B1 & $0.5 \mathrm{mg}$ \\
Vitamin B2 & $0.5 \mathrm{mg}$ \\
Vitamin B6 & $0.5 \mathrm{mg}$ \\
Vitamin B12 & $0.9 \mu \mathrm{g}$ \\
Folic Acid & $90 \mu \mathrm{g}$ \\
Niacin & $6 \mathrm{mg}$ \\
Iron (Fumarate) & $10 \mathrm{mg}$ \\
Zinc & $4.1 \mathrm{mg}$ \\
Copper & $0.56 \mathrm{mg}$ \\
Selenium & $17 \mu \mathrm{g}$ \\
lodine & $90 \mu \mathrm{g}$ \\
\hline
\end{tabular}

Source: [26]

$D R I$ Dietary reference intakes

\section{Follow up and outcomes assessment}

Two trained licensed nurses assessed infants at the clinics at baseline, 3-month intervals through the supplementation period (12 months), and 3 months after the end of supplementation. At baseline, information on socio-demographic, nutritional status, and health status of infants and their parents was gathered. At each visit, mothers were also interviewed about the general health status of infants and occurrence of adverse events such as vomiting, diarrhea, constipation, and discomfort. Based on a feasibility study undertaken by the author before the current study, compliance and acceptance of MNP as well as preference for at-home foods mixed with MNP were shown to be adequate [30].

The two nurses performed anthropometric assessments (every 3 months) using standard procedures [31] including body weight and length. Anthropometric measurements were taken in duplicate and were then averaged for each participant. The WHO ANTHRO program was used to calculate the $\mathrm{z}$ scores, and then the results were compared to the 2006 WHO growth reference [32]. Capillary blood (finger or heel prick) hemoglobin was measured at baseline, end of supplementation, and 3 months after the end of supplementation by the laboratory technician of the UNRWA clinic following the standard procedures established by the WHO [33] using spectrophotometer. Intakes of energy and micronutrients were assessed using a 1 day of 24-h diet recall at baseline, 6 months of supplementation, and at the end of supplementation period and the findings were compared to the EARs of under-three children to assess adequacy [26]. Nutritional data were analyzed by Nutritionist Pro ${ }^{\mathrm{Tm}}$ software version 7.1.0 (Axxya Systems, USA).

\section{Statistical analysis}

For all statistical tests, analysis was based on intention to treat; that is, the study results were analyzed using all available data on participants regardless of any protocol violation, such as imperfect adherence or contamination of treatment. Skewness and kurtosis values confirmed that data was normally distributed. The baseline comparison between the groups was done using the student's t test. The Chi-square test was used to examine the association between two categorical variables. Repeatedmeasures analysis of variance (RMANOVA) was used to test for changes in growth, hemoglobin concentration, and energy and micronutrient intakes over time within and between groups, followed by Bonferroni correction for multiple comparisons. Subsequently, data were analyzed using analysis of covariance (ANCOVA) to adjust for sex. The results were considered significant when $p \leq 0.05$. Practical clinical significant differences were determined by the effect size measured by the Standardized Mean Difference (SMD) ( small $=0.2$; medium $=0.5$; large $=0.8$ ). The SPSS software, version 23 (SPSS Inc., Chicago, IL) was used for the statistical analysis.

\section{Results}

A total of 170 infants completed the supplementations (82 experimental group, 88 control group). The participation rate at the end of the study was $85 \%$ and mean overall compliance was $91.17 \%$ of the total intended MNP dose (empty/used MNP sachets were counted on monthly basis). There were no significant differences in baseline measurements between the children who completed the study and those who did not, as well as, no significant differences in feeding practices between the

Table 2 Composition and dosage of the NMS

\begin{tabular}{llll}
\hline Micronutrient & Composition & Onset (old) & Duration \\
\hline Vitamin A & $300-600 \mu \mathrm{g}$ & 9 months & One squeezed capsule repeated every 6 months. \\
Vitamin D & $10 \mu \mathrm{g}$ & 9 months & 2 drops for 30 days and repeated every 3 months. \\
Iron (Sulphate) & $2 \mathrm{mg} / \mathrm{kg} /$ day & 6 months & 2 drops for 12 months. \\
\hline
\end{tabular}


two groups were reported throughout the study period (Table 3). No adverse events were observed among participants throughout the study. As shown in Table 4, the baseline characteristics of the participants showed no significant difference between the two groups and all of participants had normal birth weight. However, approximately, more than half of infants did not meet the EAR for iron, vitamin $\mathrm{A}$, and vitamin D intakes.

\section{Hemoglobin concentration}

The hemoglobin concentrations in the two groups through the 15-month study period (12 months of supplementation and 3 months after the end of supplementation) are presented in Table 5. Over the study period, hemoglobin concentration reduced significantly in the two groups as compared to baseline $(p<0.05)$ and the reduction was significantly greater in the control group than that in the experimental group ( 0.29 vs. 0.52 at month 12 and 0.18 vs. 0.63 at month 15). Further, in the experimental group, hemoglobin concentration increased significantly in month 15 when compared to month $12(p=0.001)$, while it continued to decrease non-significantly in the control group for the same time period. A significant difference $(p<0.05)$ between the two groups was only observed in the mean hemoglobin at the end of study (month 15) with a significant group by month of assessment interaction $(p<0.001)$. After adjustment for sex, blood hemoglobin at 12 months differed significantly between the two groups, and the experimental group has significantly greater values than did the control group.

Although the recruited infants were non-anemic at baseline, the prevalence of anemia was significantly different between the two groups at 12 months $(p<0.05)$ and 15 months $(p<0.001)$. About 29 and $18 \%$ of children in the experimental group and 54 and $52 \%$ in the control group had mild to moderate anemia at 12 and 15 months of the study, respectively (Table 6). Moreover, a significant $(p=0.001)$ and large effect size $(0.84)$ was found for the provision of MNP on hemoglobin concentration based on the SMD.

\section{Anthropometric measures}

The mean anthropometric values obtained during the study period (12 months of supplementation and 3 months after the end of supplementation) are presented in Table 5. All anthropometric indices increased significantly $(p<0.05)$ in the two groups from baseline to month 12 and 15, except for LAZ in the experimental and control groups, in addition to WAZ and weight-forlength $\mathrm{z}$ score (WLZ) in the control group. As compared to baseline, LAZ in the experimental group and WAZ, and WLZ in the control group were non-significantly increased and decreased at month 12 and 15, respectively $(p>0.05)$. Further, LAZ worsened significantly from baseline to month 12 and 15 in the control group $(p<$ 0.05). The increment in weight, length, WAZ, and WLZ in the experimental group was significantly higher than that in the control group at 12 and 15 months as compared to baseline. Although LAZ increment in the experimental group was greater than that in the control group at 12 and 15 months, it did not reach a level of significance. Significant differences were observed between the two groups in the mean anthropometric measures $(p<0.05)$ with a significant group by month of assessment interaction $(p<0.01)$. After adjustment for sex, anthropometric measures at 12 months differed significantly between the two groups, and the experimental group has significantly greater values than did the control group (Table 5).

Although all infants had normal weight-for-age, and length-for-age $\mathrm{z}$ scores at the beginning of the study, the prevalence of underweight and wasting in the control group was insignificantly higher than that in the experimental group at month 12 and 15 [underweight; (5\% vs. $0 \%)$ and ( $3 \%$ vs. $0 \%)$, wasting; $(2 \%$ vs. $0 \%)$ and $(2 \%$ vs. $0 \%)$, respectively]. Furthermore, a significant higher prevalence of stunting $(p<0.05)$ was observed in the experimental group than that in control groups at 12 months ( $0 \%$ vs. $9 \%)$ and 15 months (1\% vs. $11 \%)$, respectively (Table 6). Moreover, the findings of the current study showed a significant $(p<0.05)$ and medium to large effect size for provision of MNP on participants' weight-for-age ( $S M D=1.07$ ), length-for-age $(\mathrm{SMD}=0.95)$, and weight-for-length $(\mathrm{SMD}=0.49)$.

\section{Energy and micronutrient intakes}

Table 7 showed the changes in mean energy and micronutrients (NMS and MNP doses were excluded) intakes during the supplementation period. As compared to baseline values, mean values for energy, vitamin $\mathrm{A}$, and vitamin $\mathrm{D}$ intakes increased significantly in the two groups $(p<0.05)$. However, iron intake decreased significantly in the two groups as compared to the baseline intake $(p<0.05)$. Significant differences between the two groups were only observed in the mean vitamin $\mathrm{A}$ and vitamin D intakes after 6 months of supplementation with a significant group by month of assessment interaction for vitamin D intake $(p<0.05)$. Similar findings were also reported after adjustment for sex at 12 months.

\section{Discussion}

The first 2 years of life is a crucially important period, and adequate nutrition through this time is fundamental for growth and development of child to its fullest potential [34]. Studies have demonstrated adverse consequences of inappropriate dietary intake and practices on 
Table 3 Feeding practices at baseline and throughout the study period $(n=200)$

\begin{tabular}{|c|c|c|c|c|}
\hline Variables & $\begin{array}{l}\text { Experimental Group }(n=100) \\
\mathrm{n}(\%)\end{array}$ & $\begin{array}{l}\text { Control group }(n=100) \\
n(\%)\end{array}$ & $t / x^{2}$ value & $P$ value ${ }^{a}$ \\
\hline \multicolumn{5}{|l|}{ Type of milk } \\
\hline \multicolumn{5}{|c|}{ Exclusive breast feeding (month old) } \\
\hline 0 to 5.99 & $100(100)$ & $100(100)$ & -0.63 & 0.53 \\
\hline Mean \pm SD & $4.51 \pm 0.56$ & $4.65 \pm 0.59$ & & \\
\hline \multicolumn{5}{|c|}{ Still breastfeeding (month old) } \\
\hline At 5.99 & $90(90)$ & $96(96)$ & 2.76 & 0.09 \\
\hline At 11.99 & $68(68)$ & $64(64)$ & 0.35 & 0.65 \\
\hline At 17.99 & $4(4)$ & $3(3)$ & & $1+$ \\
\hline Mean \pm SD & $11.57 \pm 3.35$ & $11.76 \pm 2.19$ & -0.43 & 0.66 \\
\hline \multicolumn{5}{|c|}{ Infant formula (month old) } \\
\hline Birth -5.99 & $67(67)$ & $70(70)$ & 0.20 & 0.64 \\
\hline $6-11.99$ & $71(71)$ & $76(76)$ & 0.64 & 0.42 \\
\hline $12-17.99$ & $88(88)$ & $81(81)$ & 1.87 & 0.17 \\
\hline \multicolumn{5}{|l|}{ Fresh milk (month old) } \\
\hline Birth -5.99 & $60(60)$ & $56(56)$ & 0.32 & 0.56 \\
\hline $6-11.99$ & $47(47)$ & $50(50)$ & 0.18 & 0.67 \\
\hline $12-17.99$ & $87(87)$ & $93(93)$ & 2 & 0.15 \\
\hline \multicolumn{5}{|c|}{ Milk powder (other than infant formula) (month old) } \\
\hline Birth -5.99 & $7(7)$ & $5(5)$ & 0.35 & 0.55 \\
\hline $6-11.99$ & $67(67)$ & $55(55)$ & 3.02 & 0.08 \\
\hline $12-17.99$ & $83(83)$ & $90(90)$ & 2.09 & 0.14 \\
\hline \multicolumn{5}{|l|}{ Others (month old) } \\
\hline Birth -5.99 & $1(1)$ & $0(0)$ & & $1+$ \\
\hline $6-11.99$ & $0(0)$ & $1(1)$ & & $1+$ \\
\hline $12-17.99$ & $11(11)$ & $14(14)$ & 0.41 & 0.52 \\
\hline \multicolumn{5}{|c|}{ Age at introduction of complementary foods } \\
\hline \multicolumn{5}{|l|}{ Foods } \\
\hline Commercial cereals & $\begin{array}{l}65(65) \\
6.25 \pm 0.79\end{array}$ & $\begin{array}{l}68(68) \\
6.05 \pm 0.84\end{array}$ & 1.30 & 0.19 \\
\hline Porridge/Rice & $\begin{array}{l}52(52) \\
6.61 \pm 0.96\end{array}$ & $\begin{array}{l}55(55) \\
6.77 \pm 1.01\end{array}$ & -0.76 & 0.44 \\
\hline Biscuits/Breads & $\begin{array}{l}100(100) \\
5.59 \pm 0.81\end{array}$ & $\begin{array}{l}100(100) \\
5.79 \pm 0.84\end{array}$ & -1.55 & 0.12 \\
\hline Hen egg & $\begin{array}{l}63(63) \\
8.90 \pm 0.6\end{array}$ & $\begin{array}{l}69(69) \\
9.02 \pm 0.70\end{array}$ & -0.91 & 0.36 \\
\hline Meat/Fish & $\begin{array}{l}30(30) \\
12.28 \pm 1.43\end{array}$ & $\begin{array}{l}35(35) \\
12.08 \pm 1.33\end{array}$ & 0.53 & 0.59 \\
\hline Vegetables & $\begin{array}{l}90(90) \\
6.89 \pm 1.27\end{array}$ & $\begin{array}{l}89(89) \\
7.05 \pm 1.34\end{array}$ & -0.71 & 0.47 \\
\hline Fruits & $\begin{array}{l}100(100) \\
7.48 \pm 1.43\end{array}$ & $\begin{array}{l}100(100) \\
7.35 \pm 1.42\end{array}$ & 0.56 & 0.57 \\
\hline \multicolumn{5}{|l|}{ Drinks } \\
\hline Water with sugar & $\begin{array}{l}52(52) \\
4.10 \pm 0.62\end{array}$ & $\begin{array}{l}58(58) \\
4.14 \pm 0.61\end{array}$ & -0.33 & 0.74 \\
\hline Fruit juice & $\begin{array}{l}75(75) \\
5.44 \pm 0.85\end{array}$ & $\begin{array}{l}77(77) \\
5.18 \pm 0.74\end{array}$ & 1.84 & 0.06 \\
\hline Tea & $\begin{array}{l}35(35) \\
5.63 \pm 0.84\end{array}$ & $\begin{array}{l}41(41) \\
5.50 \pm 0.74\end{array}$ & 0.69 & 0.48 \\
\hline
\end{tabular}


Table 3 Feeding practices at baseline and throughout the study period $(n=200)$ (Continued)

\begin{tabular}{|c|c|c|c|c|}
\hline Variables & $\begin{array}{l}\text { Experimental Group }(n=100) \\
n(\%)\end{array}$ & $\begin{array}{l}\text { Control group }(n=100) \\
n(\%)\end{array}$ & $t / X^{2}$ value & $P$ value $^{a}$ \\
\hline Coffee & $\begin{array}{l}2(2) \\
12.5 \pm 1.41\end{array}$ & $\begin{array}{l}1(1) \\
12 \pm 0.00\end{array}$ & 0.28 & 0.82 \\
\hline Others & $\begin{array}{l}99(99) \\
8.80 \pm 0.94\end{array}$ & $\begin{array}{l}96(96) \\
8.87 \pm 0.95\end{array}$ & -0.46 & 0.64 \\
\hline
\end{tabular}

${ }^{a}$ Statistical testing using independent samples t-test or chi-square test

${ }^{\dagger}$ Fisher's exact test

*Difference is significant at $p<0.05$ (2-tailed)

**Difference is significant at $p<0.01$ (2-tailed)

Table 4 Baseline characteristics of the participated infants in the two groups ${ }^{b}$

\section{Characteristics \\ Household characteristics}

Family size (n)

Family income (NIS) ${ }^{\mathrm{c}}$

Number of under-five children (n)

Maternal characteristics

Age (years)
Body mass index
Mother's education (years)
Mother's working status (\%)
Working
Housewives
Infants' characteristics
Age (months)
Gender (\%)
Male
Female
Birth weight (kg)
Gestational age (weeks)
Infant currently breastfeeding (\%)

\section{Anthropometric measures}

$\begin{array}{lc}\text { Weight }(\mathrm{kg}) & 7.57 \pm 0.79 \\ \text { Length }(\mathrm{cm}) & 66.20 \pm 1.87 \\ \text { Weight-for-age (z score) } & -0.12 \pm 0.92 \\ \text { Length-for-age (z score) } & -0.25 \pm 0.85 \\ \text { Weight-for-length (z score) } & 0.11 \pm 1.01\end{array}$

Biochemical measure

Blood hemoglobin (g/dl)

$11.42 \pm 0.35$

$5 \pm 1.77$

0.33

$1275 \pm 818.06$

$2.01 \pm 0.76$

$1000 \pm 724.31$

0.07

$2 \pm 0.52$

0.18

$28 . .41 \pm 5.86$

$28.4 \pm 5.10$

0.70

$26.54 \pm 5.33$

$26.64 \pm 4.36$

0.19

$13.18 \pm 2.89$

$12 \pm 3.01$

0.54

0.07

6 (6)

15 (15)

85 (85)

$6.28 \pm 0.10$

$6.24 \pm 0.09$

0.08

52 (52)

48 (48)

$3.44 \pm 0.33$

$38.31 \pm 0.71$

92 (92)

.

51 (51)

49 (49)

$3.41 \pm 0.32$

0.55

$38.37 \pm 0.68$

0.59

96 (96)

$7.65 \pm 0.72$

0.47

$66.11 \pm 1.92$

0.75

$0.01 \pm 0.93$

0.47

$-0.28 \pm 0.97$

0.79

$0.28 \pm 1.25$

Energy and micronutrient intakes

$\begin{array}{ll}\text { Total energy (kcal/day) } & 656 \pm 164 \\ \text { Iron (mg/day) } & 7.51 \pm 7.40 \\ \text { Vitamin A (mcg/day) } & 299.70 \pm 120.31 \\ \text { Vitamin D (mcg/day) } & 3.24 \pm 2.6\end{array}$

*Difference is significant at $p<0.05$ (2-tailed)

${ }^{a}$ Statistical testing using independent samples t-test or chi-square test

${ }^{b}$ Values are reported as means $\pm S D$

${ }^{c}$ NIS New Israeli Shekel 
Table 5 Changes in anthropometric and biochemical measures by intervention group ${ }^{1}$

\begin{tabular}{|c|c|c|c|c|c|c|c|c|}
\hline \multirow[t]{2}{*}{ Variables } & \multicolumn{3}{|c|}{ Experimental group $(n=100)$} & \multicolumn{3}{|c|}{ Control group $(n=100)$} & \multirow{2}{*}{$\begin{array}{l}\text { Unadjusted } \\
\mathrm{p} \mathrm{Value}^{\mathrm{a}}\end{array}$} & \multirow{2}{*}{$\begin{array}{l}\text { Adjusted } \\
p \text { value }^{2}\end{array}$} \\
\hline & Baseline & 12 months & 15 months & Baseline & 12 months & 15 months & & \\
\hline \multicolumn{9}{|l|}{ Blood hemoglobin } \\
\hline $\begin{array}{l}\text { Blood hemoglobin } \\
(\mathrm{g} / \mathrm{dl})\end{array}$ & $11.42 \pm 0.35$ & $11.13 \pm 0.52^{* *}$ & $11.24 \pm 0.44^{*} \neq c$ & $11.44 \pm 0.37$ & $10.92 \pm 0.61^{* *}$ & $10.81 \pm 0.59^{* * c}$ & $0.000^{* *}$ & $0.02^{*}$ \\
\hline \multicolumn{9}{|c|}{ Anthropometric measures } \\
\hline Weight (kg) & $7.57 \pm 0.79$ & $11.31 \pm 0.95^{* * \mathrm{~b}}$ & $12.04 \pm 0.95^{* * c}$ & $7.65 \pm 0.72$ & $10.69 \pm 0.82^{* * b}$ & $11.05 \pm 0.80^{* * c}$ & $0.000^{* *}$ & $0.004^{*}$ \\
\hline Length $(\mathrm{cm})$ & $66.20 \pm 1.87$ & $81.50 \pm 2.56^{* * b}$ & $84.16 \pm 1.99^{* * \mathrm{c}}$ & $66.11 \pm 1.92$ & $79.93 \pm 1.94^{* * b}$ & $82.12 \pm 1.69^{* * c}$ & $0.000^{* *}$ & $0.01^{*}$ \\
\hline WAZ (z score) & $-0.12 \pm 0.92$ & $0.51 \pm 0.72^{* * b}$ & $0.57 \pm 0.65^{* * c}$ & $0.01 \pm 0.93$ & $0.04 \pm 0.75^{b}$ & $-0.15 \pm .0 .70^{c}$ & $0.000^{* *}$ & $0.003^{*}$ \\
\hline LAZ (z score) & $-0.25 \pm 0.85$ & $-0.01 \pm 0.92^{b}$ & $-0.21 \pm 1.33^{c}$ & $-0.28 \pm 0.97$ & $-0.57 \pm 0.81^{* b}$ & $-0.78 \pm 0.66^{* * c}$ & $0.001^{*}$ & $0.04^{*}$ \\
\hline WLZ (z score) & $0.11 \pm 1.01$ & $0.71 \pm 0.80^{* * b}$ & $1 \pm 1.79 * c$ & $0.28 \pm 1.25$ & $0.42 \pm 0.93^{b}$ & $0.31 \pm 0.88^{c}$ & $0.001^{*}$ & $0.02^{*}$ \\
\hline
\end{tabular}

${ }^{1}$ values are mean \pm SD

a Undjusted $p$ values are group $\mathrm{x}$ time of assessment interaction and were obtained by repeated measures ANOVA analysis. Experimental group, MNP and NMS; Control group, NMS only. WAZ Weight-for-age z score, LAZ Length-for-age z score, and WLZ Weight-for-length z score

Difference between groups at same time point is significant (at the 0.05 level) when letters are same

* Significantly different by paired t-test between baseline and selected time-point in the same intervention group at the 0.05 level

** Significantly different by paired t-test between baseline and selected time-point in the same intervention group at the 0.001 level

₹ Significantly different by paired t test between month 12 and 15 in the same intervention group at the 0.05 level

${ }^{2} p$ value after adjusting for sex (Model 1 ) at 12 months, groups were compared using one-way ANCOVA

nutritional status of infants and children [35, 36]. The present study examined the effect of MNP (3 sachets/ week) combined with NMS administered for a 12-month period on the nutritional status of Palestinian infants.

Although hemoglobin concentration worsened significantly in the two groups as compared to baseline, the present study showed that hemoglobin concentration was significantly higher in the experimental group than that in the control group at 3 months after the end of supplementation, and a significant increase in mean hemoglobin at the end of the study was reported in the experimental group when compared with 12 months of the supplementation. Moreover, the experimental group had a significantly lower prevalence of anemia throughout the study as compared to the control group.

In a comprehensive review of nutrition situation of Gaza Strip, mothers reported differences in frequency and amount of micronutrient drops given, highlighting poor compliance [37]. Therefore, although the absorption of iron from the ferrous sulphate used in the NMS is 2-3 times greater than the ferrous fumarate used in the MNP [38], low compliance to the NMS in Gaza Strip could explain the worst hemoglobin change in the control group. In contrast, the non-significant change in hemoglobin throughout the intervention period in the experimental group could be explained by the inclusion of zinc with iron in the MNP which could have reduced absorption due to zinc and iron interaction and may affect the results [39]. Consistent with a 12-month cluster randomized community trial in Pakistan [18]; the 6months aged non-anemic children who had iron intake less than recommended were supplemented with MNP with $10 \mathrm{mg}$ zinc, MNP without zinc or placebo daily for 12 months showed a significant decrease in the mean hemoglobin concentration between the experimental and control groups, and the decrease in hemoglobin concentration in the control group $(0.7 \mathrm{~g} / \mathrm{dl})$ was more than that in the experimental groups $(0.3$ to $0.4 \mathrm{~g} / \mathrm{dl})$. It should be pointed out that $55 \%$ of infants in this study had iron intake less than the EAR at baseline, and a significant correlation was reported between iron intake of children at 18 months old and hemoglobin concentration at 21 months old $(p=0.01)$. Furthermore, the iron dose used in the current study was lower than the WHO recommended dose of $12.5 \mathrm{mg} /$ day for children under two [40] due to the concern that higher doses of iron supplements consumed for 12 months could produce adverse effects [41]; however, no adverse event was recorded.

Nevertheless, a significant large effect size for addition of MNP on hemoglobin concentration was reported in the present study. Similarly, a significant small to large effect size were reported in a review study $(S M D=0.98)$ [42] and a meta-analysis $(\mathrm{SMD}=0.39)$ [43]. Factors such as age (younger vs. older children) and population (lowincome vs. high-income population) groups, duration of supplementation and dosage of micronutrients could explain the different outcomes, suggesting that micronutrients supplementation would have a greater effect if a well-established dosage and length of supplementation was considered among young children.

Administration of MNP three sachets per week for 12 months with NMS resulted in an overall improvement of most anthropometric measures and lower undernutrition prevalence than use of NMS alone. This supports the results of other micronutrients supplementation trials which suggest that MNP supplement was effective for improving anthropometric indices and underweight, stunting, and wasting prevalence $[13,18]$. The limited 
Table 6 The prevalence of anemia, underweight, stunting, and wasting during the study period

\begin{tabular}{|c|c|c|c|c|c|c|}
\hline \multirow[t]{3}{*}{ Clinical indicators } & \multicolumn{6}{|c|}{ Experimental $(n=100)$ and control groups $(n=100)$} \\
\hline & \multicolumn{3}{|l|}{ Month 12} & \multicolumn{3}{|l|}{ Month 15} \\
\hline & $\begin{array}{l}\text { Exp. group } \\
\mathrm{n}(\%)\end{array}$ & $\begin{array}{l}\text { Control group } \\
\mathrm{n}(\%)\end{array}$ & $\begin{array}{l}\text { Total } \\
\text { n (\%) }\end{array}$ & $\begin{array}{l}\text { Exp. group } \\
\text { n (\%) }\end{array}$ & $\begin{array}{l}\text { Control group } \\
\text { n (\%) }\end{array}$ & $\begin{array}{l}\text { Total } \\
\text { n (\%) }\end{array}$ \\
\hline \multicolumn{7}{|l|}{ Blood hemoglobin level (g/dl) } \\
\hline Normal blood hemoglobin ${ }^{a}$ & $71(71)$ & $46(46)$ & $117(58.5)$ & $82(82)$ & $48(48)$ & $130(65)$ \\
\hline Mild anemia ${ }^{\mathrm{b}}$ & $28(28)$ & $48(48)$ & $76(38)$ & $18(18)$ & $43(43)$ & $61(30.5)$ \\
\hline Moderate anemia $^{c}$ & $1(1)$ & $6(6)$ & $7(3.5)$ & $0(0)$ & $9(9)$ & $9(4.5)$ \\
\hline Severe anemia $^{d}$ & $0(0)$ & $0(0)$ & $0(0)$ & $0(0)$ & $0(0)$ & $0(0)$ \\
\hline Fisher's exact test value & & & 10.74 & & & 24.20 \\
\hline$p$ value & & & $0.003^{*}$ & & & $<0.001^{* *}$ \\
\hline \multicolumn{7}{|l|}{ Weight for age (z score) } \\
\hline Normal weight for age $\mathrm{e}^{\mathrm{e}}$ & $100(100)$ & $95(95)$ & $195(97.5)$ & $100(100)$ & $97(97)$ & $197(98.5)$ \\
\hline Moderate underweight ${ }^{f}$ & $0(0)$ & $5(5)$ & $5(2.5)$ & $0(0)$ & $3(3)$ & $3(1.5)$ \\
\hline Severe underweight ${ }^{9}$ & $0(0)$ & $0(0)$ & $0(0)$ & $0(0)$ & $0(0)$ & $0(0)$ \\
\hline Fisher's exact test value & & & 3.81 & & & 2.84 \\
\hline$p$ value & & & 0.12 & & & 0.24 \\
\hline \multicolumn{7}{|l|}{ Length for age ( $z$ score) } \\
\hline Normal length for age ${ }^{e}$ & $100(100)$ & $91(91)$ & $191(95.5)$ & $99(99)$ & $89(89)$ & $188(94)$ \\
\hline Moderate stunting ${ }^{f}$ & $0(0)$ & $9(9)$ & $9(4.5)$ & $1(1)$ & $11(11)$ & $12(6)$ \\
\hline Severe stunting ${ }^{g}$ & $0(0)$ & $0(0)$ & $0(0)$ & $0(0)$ & $0(0)$ & $0(0)$ \\
\hline Fisher's exact test value & & & 7.82 & & & 11.89 \\
\hline$p$ value & & & $0.007^{*}$ & & & $0.002^{*}$ \\
\hline \multicolumn{7}{|l|}{ Weight for length (z score) } \\
\hline Normal weight for length ${ }^{e}$ & $100(100)$ & $98(98)$ & $198(99)$ & $100(100)$ & $98(98)$ & $198(99)$ \\
\hline Moderate wasting ${ }^{f}$ & $0(0)$ & $2(2)$ & $2(1)$ & $0(0)$ & $2(2)$ & $2(1)$ \\
\hline Severe wasting ${ }^{g}$ & $0(0)$ & $0(0)$ & $0(0)$ & $0(0)$ & $0(0)$ & $0(0)$ \\
\hline Fisher's exact test value & & & 1.88 & & & 1.88 \\
\hline$p$ value & & & 0.49 & & & 0.49 \\
\hline
\end{tabular}

NB At baseline, the prevalence of anemia, underweight, stunting, and wasting was zero

${ }^{*} p<0.05$; ${ }^{* *} p<0.001$ using Fisher's exact test

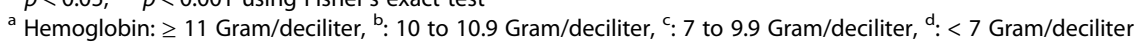

e Normal underweight, stunting, or wasting: $\geq-2$ SD of the WHO median for WAZ, LAZ, or WLZ

${ }^{f}$ Moderate underweight, stunting, or wasting: $\geq-3$ SD but $<-2$ SD of the WHO median for WAZ, LAZ, or WLZ

${ }^{g}$ Severe underweight, stunting, or wasting: $<-3$ SD of the WHO median for WAZ, LAZ, or WLZ

Table 7 Changes in energy, iron, vitamin A, and vitamin D intakes by intervention group ${ }^{1}$

\begin{tabular}{|c|c|c|c|c|c|c|c|c|}
\hline \multirow[t]{2}{*}{ Dietary factors } & \multicolumn{3}{|c|}{ Experimental group $(n=100)$} & \multicolumn{3}{|c|}{ Control group $(n=100)$} & \multirow{2}{*}{$\begin{array}{l}\text { Unadjusted } \\
p \text { value }^{\mathrm{a}}\end{array}$} & \multirow{2}{*}{$\begin{array}{l}\text { Adjusted } \\
p \text { value }{ }^{2}\end{array}$} \\
\hline & Baseline & 6 months & 12 months & Baseline & 6 months & 12 months & & \\
\hline Energy (kcal) & $655 \pm 164$ & $784 \pm 163^{* *}$ & $818 \pm 111^{* *}$ & $696 \pm 167$ & $751 \pm 114$ & $835 \pm 138^{* *}$ & 0.06 & 0.21 \\
\hline Iron (mg) & $7.51 \pm 7.40$ & $5.96 \pm 7.31$ & $3.35 \pm 1.94^{* *}$ & $7.90 \pm 8.55$ & $5.84 \pm 5.18$ & $3.28 \pm 1.66^{* *}$ & 0.88 & 0.61 \\
\hline $\begin{array}{l}\text { Vitamin A } \\
\text { (RAE, mcg) }\end{array}$ & $299.7 \pm 120.3$ & $409.7 \pm 297.2^{* b}$ & $362.37 \pm 119.08^{*}$ & $264.32 \pm 161.1$ & $340.04 \pm 114.80^{*} b$ & $367.71 \pm 224.24^{*}$ & 0.15 & 0.32 \\
\hline Vitamin D (mcg) & $3.24 \pm 2.60$ & $5.19 \pm 3.62^{* c}$ & $6.97 \pm 3.44^{* *}$ & $3.85 \pm 3.03$ & $6.98 \pm 3.27^{* * c}$ & $6.45 \pm 2.67^{* *}$ & $0.003^{*}$ & $0.02^{*}$ \\
\hline
\end{tabular}

${ }^{1}$ values are mean $\pm \mathrm{SD}$

${ }^{a} p$ values are group $\mathrm{x}$ time of assessment interaction and were obtained by repeated measures ANOVA analysis

Experimental group, MNP and NMS; Control group, NMS only

Difference between groups at same time point is significant (at the 0.05 level) when letters are same

* Significantly different by paired t-test between baseline and selected time-point in the same intervention group at the 0.05 level

** Significantly different by paired t-test between baseline and selected time-point in the same intervention group at the 0.001 level

${ }^{2} p$ value after adjusting for sex (Model 1) at 12 months, groups were compared using one-way ANCOVA 
age range of children in the present study (6 to 18 months), and high compliance to supplementation regimen which was satisfactory (91\%) could contribute to the observed improvement. Rivera et al. indicated that the supplementation effect with high compliance rate $86 \%$ on length gain during the first years of life demonstrated the highest benefit [44]. The greater effect on young children (less than 2 years) than older children is biologically plausible. Under-two children grow at a faster rate than older children, their micronutrient needs to sustain this accelerated growth are greater, and their diets are often more restricted than those of older children. Therefore, most of the supplementary feeding effect occurs during the first 2 years of life [45]. However, the findings of meta-analysis to assess the effect of fortification of home food with micronutrient powders on the nutritional status of healthy children aged 6 to 23 months did not report an impact on growth. The possible rationale for difference between results could be due to different formulations of the micronutrient powders, different duration of supplementation (ranged from 2 to 12 months), different compliance rate, and some of the children were apparently healthy but at risk of having a highly prevalence of diseases including malaria, diarrhea or other undernutrition forms [21].

In term of effect size, a significant medium to large effect size for the provision of MNP on weight-for-age, length-for-age, and weight-for-length was reported in the current study. In the line of this study, a metaanalysis including 16 micronutrients supplementation studies (5 anemia treatment trials, and 11 prevention trials and duration from 2 to 13 months) showed a significant small to medium effect size for MNP with small amount of energy on weight-for-age $(\mathrm{SMD}=0.39)$, length/height-for-age (SMD =0.41), and weight-forlength $(\mathrm{SMD}=0.12)$. However, a non-significant small effect size for MNP without energy on weight-for-age $(\mathrm{SMD}=0.08)$, length/height-for-age $(\mathrm{SMD}=0.02)$, and weight-for-length $(\mathrm{SMD}=0.01)$ was reported in the same review [46]. Few studies are available for the effect size comparison [46], but these findings supported the contention that home fortification with supplements that include some energy would be more effective particularly among unhealthy or highly risk children and programs with short duration regimens.

\section{Limitations of the study}

The present study had several limitations, including inability to use placebo or keeping participants blind to the type of supplement due to logistical, and social constrains, respectively. However, outcomes being measured were objective and duplicate assessment of outcomes was considered. Although attrition could have introduced selection bias, there was no significant difference between children who remained in the study and those who dropped out which dismissed that possibility. Finally, the present study used a rigorous design (randomized controlled trial) with many potential confounders were assessed as well as intention to treat analysis.

\section{Conclusion}

The results of the present study suggest that starting MNP supplements at the age of 6 months, when human milk alone fails to meet the dietary requirements and complementary foods of poor nutritional value are introduced, may be promising in improving the nutritional status during the complementary feeding period and thereafter. Particularly, in infants at risk of undernutrition living in developing countries such as Palestine, where common complementary foods are poor in nutritive value and the NMS program distributed by the Ministry of Health is inadequate. In addition, micronutrient powder demonstrated a prophylactic effect in the present study which suggested that modifying the dose or/and the duration of MNP intake may have possible effects on initially undernourished children, but further studies are required.

\section{Acknowledgments \\ The authors thank the participants and their parents for their involvement in the study and the UNRWA team members for their assistance and cooperation throughout the study. \\ Authors' contributions \\ All authors have contributed to conception, design, and acquisition of this study. As well as they have contributed to preparation of the manuscript and approved the final draft of manuscript for submission to Nutrition Journal. Ali Albelbeisi and Zalilah Shariff were contributed to analysis, interpretation of results, and critically revised the manuscript.}

\section{Funding}

None.

\section{Availability of data and materials} Not Applicable.

\section{Ethics approval and consent to participate}

The study was approved by the Helsinki Ethics Committee of Gaza Strip, as well as the Ethics Committee for Research Involving Human Subjects (JKEUPM) of the Faculty of Medicine and Health Sciences, Universiti Putra Malaysia. Permission to conduct the study in the UNRWA clinics was obtained from the Health Affairs Center of The United Nations Relief and Works Agency for Palestine Refugees in the Near East (UNRWA) in Gaza Strip.

Consent for publication

Written informed consent was obtained from the participants for publication of this work.

\section{Competing interests}

The authors declare that they have no competing interests.

\section{Author details}

${ }^{1}$ Department of Nutrition and Dietetics, Faculty of Medicine and Health Sciences, University Putra Malaysia, Seri Kembangan, Malaysia. ${ }^{2}$ Department of Community Health, Faculty of Medicine and Health Sciences, University Putra Malaysia, Seri Kembangan, Malaysia. ${ }^{3}$ Faculty of Public Health, Al Quds University of Gaza- Palestine, Jerusalem, Palestinian Territory. 
Received: 8 January 2020 Accepted: 27 November 2020

Published online: 05 December 2020

\section{References}

1. Micronutrient Initiative. Investing in the future: a united call to action on vitamin and mineral deficiencies. Ottawa: Micronutrient Initiative; 2009.

2. Tulchinsky TH. Micronutrient deficiency conditions: global health issues. Public Health Rev. 2010;32(1):243.

3. WHO. Global health risks: mortality and burden of disease attributable to selected major risks: World Health Organization; 2. https://www.who.int/ healthinfo/global_burden_disease/GlobalHealthRisks_report_full.pdf.

4. WHO. Worldwide prevalence of anaemia 1993-2005. In: WHO global database on anaemia: World Health Organization; 2008. https://www.who. int/nutrition/publications/micronutrients/anaemia_iron_deficiency/97892415 96657/en/.

5. Palestinian $\mathrm{MOH}$, Palestinian micronutrient survey, 2013. 2014

6. El Kishawi RR, et al. Anemia among children aged 2-5 years in the Gaza Strip-Palestinian: a cross sectional study. BMC Public Health. 2015;15(1):1.

7. Burchi F, Fanzo J, Frison E. The role of food and nutrition system approaches in tackling hidden hunger. Int J Environ Res Public Health. 2011; 8(2):358-73

8. Gibson RS, et al. A review of phytate, iron, zinc, and calcium concentrations in plant-based complementary foods used in low-income countries and implications for bioavailability. Food Nutr Bull. 2010:31(2 suppl2):S134-46.

9. Bhutta ZA, Salam RA, Das JK. Meeting the challenges of micronutrient malnutrition in the developing world. Br Med Bull. 2013;106(1):7-17.

10. Horton, A.H., Rivera JA. Copenhagen consensus 2008 challenge paper: hunger and malnutrition. 2008. [cited 2012 10, 2]; Available from: http:// www.copenhagenconsensus.com/Admin/Public/Download.aspx?file=Files\%2 fFiler\%2fCC08\%2fPapers\%2fOfficial+papers\%2fCopenhagen_Consensus_2 008_-_hunger_and_malnutrition.pdf.

11. Sazawal S, et al. Effects of routine prophylactic supplementation with iron and folic acid on admission to hospital and mortality in preschool children in a high malaria transmission setting: community-based, randomised, placebo-controlled trial. Lancet. 2006;367(9505):133-43.

12. UNICEF. The state of the world's children 2011: adolescence-an age of opportunity: UNICEF; 2011. https://www.unicef.org/sowc2011/pdfs/SOWC-2 011-MainReport_EN_02092011.pdf.

13. Chen $\mathrm{K}$, et al. Effects of vitamin A, vitamin A plus iron and multiple micronutrient-fortified seasoning powder on preschool children in a suburb of Chongqing, China. J Nutr Sci Vitaminol. 2008;54(6):440-7.

14. Jack SJ, et al. Effect of micronutrient sprinkles on reducing anemia: a clusterrandomized effectiveness trial. Arch Pediatr Adolesc Med. 2012;166(9):84250 .

15. Mahfuz $M$, et al. Effect of micronutrient powder supplementation for two and four months on hemoglobin level of children 6-23 months old in a slum in Dhaka: a community based observational study. BMC Nutr. 2016; 2(1):21.

16. Ramakrishnan $U$, et al. Multiple micronutrient supplementation during early childhood increases child size at 2 y of age only among high compliers. Am J Clin Nutr. 2009;89(4):1125-31.

17. Ramakrishnan $U$, Nguyen $P$, Martorell R. Effects of micronutrients on growth of children under $5 \mathrm{y}$ of age: meta-analyses of single and multiple nutrient interventions. Am J Clin Nutr. 2009;89(1):191-203.

18. Soofi $\mathrm{S}$, et al. Effect of provision of daily zinc and iron with several micronutrients on growth and morbidity among young children in Pakistan: a cluster-randomised trial. Lancet. 2013;382(9886):29-40.

19. De Pee, et al. Quality criteria for micronutrient powder products: report of a meeting organized by the World Food Programme and Sprinkles Global Health Initiative. Food Nutr Bull. 2008;29(3):232-41.

20. Zlotkin $\mathrm{SH}$, et al. Micronutrient sprinkles to control childhood anaemia. PLoS Med. 2005;2(1):e1.

21. De-Regil LM, et al. Home fortification of foods with multiple micronutrient powders for health and nutrition in children under two years of age (review). Evid Based Child Health. 2013;8(1):112-201.

22. MOH. Maternal and child nutrition protocols, West bank/Gaza strip: MARAM; 2005;2:30. https://www.who.int/hac/crises/international/wbgs/oPt_Review_ of nutrition situation_June2005.pdf.

23. Radi S, Mourad TA, Papandreou C. Nutritional status of Palestinian children attending primary health care centers in Gaza. Indian J Pediatr. 2009;76(2): $163-6$.
24. UNRWA. West Bank and Gaza Strip population census. 2010 [cited 2015 3, 8]; Available from: http://www.unrwa.org/userfiles/2010012035949.pdf.

25. UNRWA. UN Relief \& Works Agency (UNRWA): Palestinian refugees in the Gaza Strip. 2013 [cited 2014 15,2]; Available from: http://www. jewishvirtuallibrary.org/jsource/UN/unrwa6.html.

26. Whitney E, Rolfes SR. Understanding nutrition. 11th ed. Belmont: Thomson Higher Education. A - C; 2008.

27. $\mathrm{MOH}$. Maternal and child nutrition protocols: MARAM: West bank/Gaza strip; 2005:34. https://www.who.int/hac/crises/international/wbgs/oPt_Review_of_ nutrition_situation_June2005.pdf.

28. Cnsumer lab. Recommended daily intakes and upper limits for nutrients. 2013 [cited 2013 18,11]; Available from: http://www.consumerlab.com/rdas/.

29. UNICEF, WFP, Home Fortification Technical Advisory Group. Home fortification with micronutrient powders (MNP). In: Sight and life: Sight and Life Magazine; 2013. p. 64. https://www.unicef.org/nutrition/files/Sight_and_ Life_MNPs.pdf.

30. Albelbeisi $A$, et al. Use of micronutrient powder in at-home foods for young children (6-18 months): a feasibility study. Pak J Nutr. 2017;16(5):372-7.

31. CDC. National Health and Nutrition Examination Survey (NHANES): anthropometry procedures manual. 2007 [cited 2013 10,11]; Available from: http://www.cdc.gov/nchs/data/nhanes/nhanes_07_08/manual_an.pdf.

32. WHO. WHO Anthro (version 3.2. 2, January 2011) and macros. Geneva: World Health Organization; 2011.

33. WHO, Manual of basic techniques for a health laboratory. 2003.

34. Beuker KT, et al. Development of early communication skills in the first two years of life. Infant Behav Dev. 2013;36(1):71-83.

35. Saha KK, et al. Appropriate infant feeding practices result in better growth of infants and young children in rural Bangladesh. Am J Clin Nutr. 2008; 87(6):1852-9.

36. Ahmed T, et al. Determinants of undernutrition in children under 2 years of age from rural Bangladesh. Indian Pediatr. 2012;49(10):821-4.

37. $\mathrm{MOH}, \mathrm{WHO}$, and UNICEF. The state of nutrition: West Bank and Gaza Strip: a comprehensive review of nutrition situation of West Bank and Gaza Strip. 2005 [cited 2014 8,2]; Available from: http://www.who.int/hac/crises/ international/wbgs/oPt_Review_of nutrition_situation_June2005.pdf.

38. Sarker SA, et al. Helicobacter pylori infection, iron absorption, and gastric acid secretion in Bangladeshi children. Am J Clin Nutr. 2004;80(1):149-53.

39. Zlotkin S, et al. Home-fortification with iron and zinc sprinkles or iron sprinkles alone successfully treats anemia in infants and young children. J Nutr. 2003;133(4):1075-80.

40. Stoltzfus RJ, Dreyfuss ML. Guidelines for the use of iron supplements to prevent and treat iron deficiency anemia, vol. 2: Ilsi Press Washington^ eDC DC; 1998. http://www.health-mall.in/files_hl/guidelines_iron_ supplementation.pdf.

41. Fraga CG, Oteiza PI. Iron toxicity and antioxidant nutrients. Toxicology. 2002; 180(1):23-32.

42. Salam RA, et al. Effectiveness of micronutrient powders (MNP) in women and children. BMC Public Health. 2013;13(3):1.

43. Allen LH, Peerson JM, Olney DK. Provision of multiple rather than two or fewer micronutrients more effectively improves growth and other outcomes in micronutrient-deficient children and adults. J Nutr. 2009;139(5): 1022-30.

44. Rivera JA, et al. Multiple micronutrient supplementation increases the growth of Mexican infants. Am J Clin Nutr. 2001;74(5):657-63.

45. Schroeder DG, et al. Age differences in the impact of nutritional supplementation on growth. J Nutr. 1995;125(suppl_4):1051S-9S.

46. Dewey KG, Yang Z, Boy E. Systematic review and meta-analysis of home fortification of complementary foods. Matern Child Nutri. 2009;5(4):283-321.

\section{Publisher's Note}

Springer Nature remains neutral with regard to jurisdictional claims in published maps and institutional affiliations. 